Gynäkologe 2005 · 38:33-38 DOI 10.1007/s00129-004-1639-z

Online publiziert: 13. Januar 2005

(c) Springer Medizin Verlag 2005

Redaktion

A. Queißer-Luft, Mainz

H. Schneider, Bern

D. Wunder · Universitätsfrauenklinik, Inselspital Bern

\title{
Fehlbildungen nach assistierter Reproduktionsmedizin
}

Sterilität selbst $[4,27,29]$ als mögliche Verursacher angesehen.

ICSI ist eine noch deutlich invasivere Technik als IVF, da ein Spermium durch die Eizellmembran injiziert (• Abb. 1) und dadurch eine Befruchtung durch ein Spermium möglich wird, welches normalerweise nie zu einer Befruchtung geführt hätte. Damit sind Gefahren durch technikabhängige und technikunabhängige Risiken möglich [30]. Technikabhängige Risiken werden gesehen in der physikalischen und biochemischen Zerstörung des Ooplamas oder sogar des Spindelapparats und in der Möglichkeit der Injektion von biochemischen Verunreinigungen. Als technikunabhängige Risiken werden die Injektion eines chromosomal abnormen Spermiums vermutet oder die Übertragung von bekannten oder unbekannten Gendefekten (wie zum Beispiel AZF-Deletionen auf dem Y-Chromosom oder ZystischeFibrose-Mutationen), welche oft Ursache der Infertilität sind.

\section{Schwierige Studienlage}

Bei der Interpretation der veröffentlichten Studien sind mehrere Punkte zu beachten. Einerseits bestehen erhebliche Unterschiede im Studiendesign und bei der Fallzahl. Andererseits führen Unterschiede in der Definition von schweren und leichten Malformationen zu erheblichen Problemen. Korrekterweise werden Malformationen als morphologische Defekte und/ oder Dysfunktion definiert, welche den Tod, eine funktionelle Behinderung oder eine chirurgische Intervention zur Folge haben. Schwere Fehlbildungen („major malformations") sind von medizinischer und/oder kosmetischer Bedeutung (Bei- spiele sind Lippen-Kiefer-Gaumenspalte, Hydrozephalus), kleinere Fehlbildungen („minor malformations“) sind von keiner oder nur geringer medizinischer und/ oder kosmetischer Bedeutung (Beispiele sind Klinodaktylie, Syndaktylie).

Aber nicht nur die Uneinheitlichkeit der Definitionen von schweren und leichten Malformationen ermöglichen bei der Auswertung ein erhebliches Bias. Die nach ICSI gezeugten Kinder werden tendenziell auch einer genaueren Untersuchung unterzogen und Fehlbildungen werden im Vergleich zu Kindern nach Spontankonzeption bevorzugt gemeldet. Außerdem beeinflusst die Ausbildung und Erfahrung des Untersuchers in hohem Maße die Fehlbildungserkennung.

Auch bezüglich Chromosomenanomalien bei Kindern nach ART verglichen mit spontan gezeugten Kindern ermöglicht die Auswertung ein erhebliches Bias. Denn es wird bei den (außerdem signifikant älteren) Müttern aus dem ART-Kollektiv im Durchschnitt öfter eine invasive pränatale Diagnostik durchgeführt als bei Frauen nach Spontanschwangerschaften [22].

Eine Umgehung von einigen der genannten statistischen Verfälschungen wäre nur durch ein Matching zwischen Kontrollpopulation und ART-Patientinnen hinsichtlich Alter, Parität, ethnischer Zugehörigkeit, Einlings-/Mehrlingsgeburten, Umgebung etc. sowie Alter des Vaters möglich. Man denke auch daran, dass einige Malformationen mit Mangelernährung oder Umwelttoxinen in Verbindung gebracht werden.

Bei der Durchsicht der Studien gilt zu beachten, was mit was verglichen wurde: IVF- gegenüber ICSI-Kinder, IVF-Kinder gegen spontan gezeugte Kinder, IC- von Eireifung und zur Ovulationsauslösung notwendig sind $[19,25,34]$ oder die 


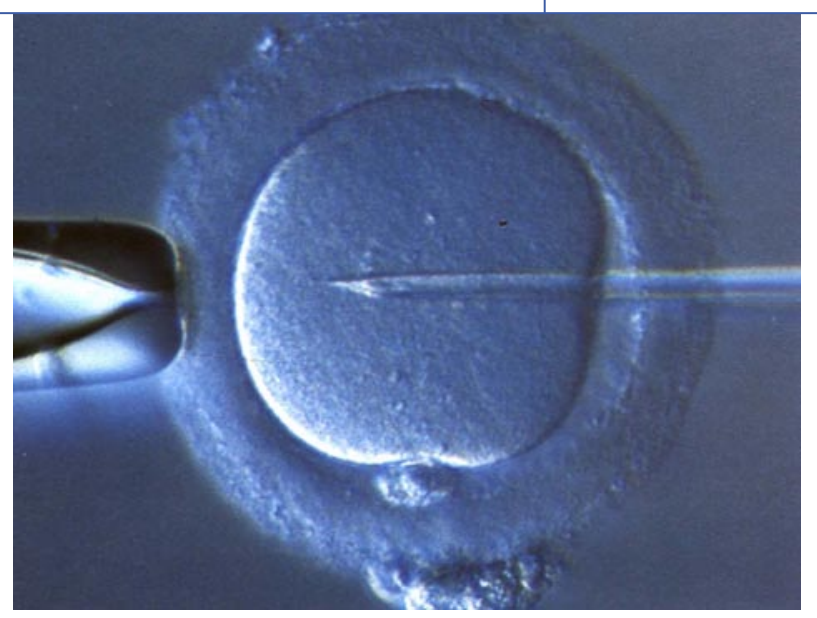

Abb. $1<$ Intrazytoplasmatische Spermieninjektion (ICSI)

SI-Kinder gegen spontan gezeugte Kinder oder IVF und ICSI-Kinder gemischt gegen spontan gezeugte Kinder. Hier ist vorwegzunehmen, dass sich die meisten Studien auf Malformationen nach ICSI beschränken und die Datenlage bezüglich Malformationen bei IVF-Kindern sehr beschränkt ist. Im Vergleich von IVF zu Spontankonzeption gibt es eigentlich nur die Daten von Hansen et al. [20], die den heutigen Ansprüchen an ein gutes Studiendesign genügen. Daraus geht hervor, dass sich das Risiko einer Malformation nach IVF nicht von dem Risiko nach ICSI unterscheidet. Paare, bei denen eine IVF vorgesehen ist, sollten nach heutiger Datenlage also gleich beraten werden wie Paare, bei denen eine ICSI vorgesehen ist.

Die folgenden Ausführungen beschränken sich auf echte „Fehlbildungen nach assistierter Reproduktionsmedizin“. Auf andere Störungen wie „niedriges Geburtsgewicht", „Schwangerschaftsverlauf“, „Frühgeburtlichkeit“ oder „Wachstum und physische, psychomotorische, sozioemotionale, kognitive und intellektuelle Entwicklung" nach künstlicher Befruchtung soll hier nicht eingegangen werden.

\section{Malformationen nach assistierter Reproduktion}

Viele frühere Studien zeigen ein identisches Fehlbildungsrisiko von IVF- verglichen mit ICSI-Schwangerschaften $[6,7$, 18]. Damals durfte aufgrund der vielen Studien der beruhigende Schluss gezogen werden, dass das Fehlbildungsrisiko sowohl von IVF-Schwangerschaften $[2,3$, $28,32,33,35]$ wie auch von ICSI-Schwangerschaften $[8,9,10,36]$ verglichen mit spontan gezeugten Kindern identisch ist. Diese Resultate blieben jedoch nicht unwidersprochen, denn mehrere neue Studien $[17,37]$ deuteten auf ein erhöhtes Risiko für Hypospadien nach ICSI hin und auch im IVF-Kollektiv zeigte sich ein erhöhtes Risiko für Malformationen [20, 23].

Die größte deutsche prospektive, kontrollierte Multizenterstudie [24] untersuchte die Fehlbildungsrate von 3372 ICSIKindern und verglich diese mit dem prospektiven Geburtenregister (Mainzer Modell). In beiden Kohorten wurden die gleichen Definitionen und Kriterien für Malformationen angewendet (EUROCAT: Europaen Registry of Congenital Anomalies and Twins; International Clearinghouse of Birth Defects Monitoring Systems). Die Fehlbildungsrate betrug 8,6\% für ICSIund 6,9\% für spontan gezeugte Kinder, RR $1,25(1,11-1,40)$. Betroffen waren vor allem Urogenitalsystem und Herz. Es fand sich kein Unterschied in Abhängigkeit vom Mehrlingsstatus, der Zahl der Spermien im Ejakulat oder der Herkunft der Spermien (ejakuliert, epidiymal oder testikulär). Letzteres fanden auch Van Steirteghem et al. [35] und Bonduelle et al. [11].

Katalinic et al. [22] untersuchte die Fehlbildungsrate von 3372 ICSI-Kindern und verglich diese mit 8016 natürlich gezeugten Kindern des Registers von SachsenAnhalt ebenfalls nach den gleichen Definitionen und Kriterien für Malformationen. Die Fehlbildungsrate betrug 8,7\% für ICSI- und 6,1\% für spontan gezeugte Kinder, RR 1,44 (1,25-1,65). Nach Korrektur für Risikofaktoren sank das relative Risiko auf $1,24(1,02-1,50)$ ab. Betroffen waren vor allem Gastrointestinaltrakt, Urogenitalsystem sowie Herz.
Erklärungen für dieses erhöhte Missbildungsrisiko sind einerseits erhöhte väterliche und mütterliche soziodemographische und anamnestische Risikofaktoren (- Tabelle 1), andererseits auch die Infertilität an und für sich [4, 27, 29]. Eine Risikoerhöhung durch die Technik selbst kann jedoch nicht ganz ausgeschlossen werden. Als Schlussfolgerungen für die Beratung von Kinderwunschpaaren ergibt sich aus diesen Studienergebnissen:

- Nach einer Spontankonzeption ist bei jeder 15. Schwangerschaft mit einer schweren Fehlbildung zu rechnen, nach assistierter Reproduktionsmedizin (IVF und ICSI) bei jeder 12.

\section{Chromosomale Aberrationen nach assistierter Reproduktion}

In den ersten Follow-up-Studien von Bonduelle et al. konnten keine Unterschiede bezüglich chromosomaler Aberrationen gefunden werden, weder im Vergleich von ICSI- mit IVF-Kindern [6] noch im Vergleich von ICSI-Kindern mit der Normalbevölkerung [10]. Allerdings zeigte eine noch im gleichen Jahr publizierte Studie von Bonduelle et al. [9] im ICSI-Kollektiv eine leicht erhöhte Rate chromosomaler De-novo-Aberrationen im Vergleich zur Normalbevölkerung. Dieses Ergebnis konnte in Folgestudien $[1,12]$ bestätigt werden und es wurde eher im Zusammenhang mit den Charakteristiken der infertilen Männer (z. B. Oligospermie) gesehen als mit der ICSI-Technik selbst.

In einer großen Folgestudie zeigten Bonduelle et al. [11] in Amniozentesen von Frauen gleichen Alters eine signifikant höhere Rate (1,6\% vs. 0,5\%) an De-novo aufgetretenen chromosomalen Aberrationen in 1586 ICSI-Schwangerschaften (bei $47 \%$ aller ICSI-Schwangerschaften wurden eine invasive pränatale Diagnostik durchgeführt). Das Risiko von 1,6\% für De-novoAnomalien nach ICSI ist gleich hoch wie das Risiko, welches eine 40-jährige Frau für De-novo-Anomalien nach Spontankonzeption hat.

Bemerkenswert ist, dass die ICSI-Kinder mit gonosomalen Anomalien und de-novostrukturellen Aberrationen bei der Geburt alle phänotypisch normal waren! 
Hätte man sie nicht karyotypisiert, hätte man die chromosomalen Aberrationen gar nicht bemerkt. Es ist jedoch bekannt, dass 5-10\% der Kinder mit de-novo-strukturellen Aberrationen Entwicklungsprobleme haben können, die sich bei der Geburt noch nicht zeigen.

Das Risiko für eine chromosomale $\mathrm{Ab}$ erration war umso höher, je schlechter die Spermienparameter (Gesamtzahl und Motilität, nicht aber Morphologie) der Väter waren. Auch andere Studienergebnisse [5, 14, 21] deuten darauf hin, dass neben dem erhöhten mütterlichen Alter väterliche genetische Anomalien in Spermatozoen wahrscheinlich eher Ursache genetischer Anomalien bei ICSI-Kindern sind als die ICSI-Technik selbst. Van Steirteghem [35] zeigte ebenfalls erhöhte De-novo gonosomale (o,6\% statt o,2\%) und strukturelle autosomale Aberrationen (o,4\% statt 0,07\%) bei ICSI-Schwangerschaften im Vergleich zur Normalbevölkerung.

Eine Übersicht der Studienergebnisse bezüglich chromosomaler Aberrationen zeigt $\bullet$ Tabelle 2 .

\section{Empfehlungen}

Ob eine Abtreibung wegen gonosomalen Aberrationen durchgeführt werden soll, wird diskutiert und debattiert, denn außer bei Turner-Patientinnen treten bei gonosomalen Aberrationen (Klinefelter, Triple XXX und XYY-Syndrom) kaum Fehlbildungen auf. Außerdem kommt es zu keiner geistigen Behinderung in diesen Fällen, wenn auch ein leicht niedrigerer Intelligenzquotient mit Lernschwierigkeiten und Probleme sozialer Adaptation beobachtet wurden [25].

Wegen der erhöhten Inzidenz von chromosomalen De-novo-Aberrationen nach ICSI empfehlen Bonduelle et al., allen Paaren, bei denen eine ICSI vorgesehen ist, als Vorabklärung eine Karyotypisierung anzubieten und eine Abklärung auf zystische Fibrose sowie Mikrodeletionen im Falle einer ausgeprägten Oligospermie oder Azoospermie des Mannes. Hierbei muss jedoch beachtet werden, dass eine Keimzellaneuploidie auch bei normalem väterlichem Karyotyp auftreten kann, da durch intratestikuläre Veränderungen nicht nur die Spermatogenese, sondern auch Kontrollmechanismen der chromosomalen Teilung während

Gynäkologe 2005 · 38:33-38

DOI 10.1007/s00129-004-1639-z

○) Springer Medizin Verlag 2005

\section{Wunder}

\section{Fehlbildungen nach assistierter Reproduktionsmedizin}

Zusammenfassung

Insgesamt deuten die Daten auf ein leicht erhöhtes Malformationsrisiko nach assistierter Reproduktionsmedizin (ART) hin. Wenn auch die Studien in ihren Ergebnissen stark schwanken und das absolute Risiko für ein Kind mit Fehlbildungen klein ist, müssen die Paare darüber aufgeklärt sein. Gesichert ist, dass Eltern nach In-vitro-Fertilisation und intrazytoplasmatischer Spermieninjektion (ICSI) durch soziodemographische und anamnestische Hintergrundsrisiken per se ein erhöhtes Risiko haben, ein Kind mit einer Fehlbildung zu zeugen. In der Kinderwunschsprechstunde müssen die Paare außerdem darüber aufgeklärt werden, dass durch ICSI gezeugte Kinder im Vergleich zum Normalkollektiv ein höheres Risiko für chromosomale Anomalien haben. Dies leitet sich aus den vererbten chromosomalen Anomalien (1-4\%) $a b$, welche schon bei den Eltern (vornehm-

lich bei den Vätern) vorhanden sind, oder durch chromosomale De-novo-Anomalien, welche vorwiegend durch die schlechte Spermienqualität (2,1\% bei einer Spermienzahl <20 Mio./ml) zustande kommen. Die Gesundheit von Kindern nach assistierter Reproduktionsmedizin muss unbedingt in weiteren Studien ausgewertet werden, um klare Schlüsse über die Sicherheit dieser Techniken zu erlangen. Immer noch unklar sind vor allem die Relevanz des Risikofaktors „Sterilität" an und für sich sowie die Bedeutung epigenetischer Veränderungen nach Einsatz der assistierten Reproduktionsmedizin.

\section{Schlüsselwörter}

In-vitro-Fertilisation · Intrazytoplasmatische Spermieninjektion - Reproduktionsmedizin . Malformationen · Chromosomale Aberrationen

\section{Malformations after assisted reproductive medicine}

\section{Abstract}

New follow-up studies indicate a slightly increased risk of malformations after assisted reproductive techniques (ART). Even if study results vary a lot and the absolute risk for congenital malformations in ART children is small, couples must be properly informed. It is undisputed that parents after in vitro festilization (IVF) or intracytoplasmatic sperm injection (ICSI) have a greater risk to father a child with malformations because of underlying sociodemographic and anamnestic risk factors. Couples have also to be counseled that ICSI-children have a greater risk for chromosomal anomalies compared with naturally conceived children. This is due to transmitted chromosomal anomalies (1-4\%), which are al- ready existent in parents (predominantly fathers), or due to de-novo chromosomal anomalies, originating mostly of bad sperm quality $(2.1 \%$ when the sperm count is $<20 \mathrm{Mio}$. $/ \mathrm{ml}$ ). The health of children after assisted reproductive medicine must be systematically documented in follow-up studies to draw clear conclusions regarding the safety of these techniques. The importance of the risk factor "sterility" itself and the significance of epigenetic changes are still unclear.

\section{Keywords} In vitro fertilization - Intracytoplasmatic sperm injection - Reproductive medicine Malformations · Chromosomal anomalies 
Tabelle 1

Soziodemographische sowie anamnestische Risikofaktoren der ICSI-Mütter und -Väter gegenüber Eltern von Spontanschwangerschaften

- Erhöhtes mütterliches Alter

- Erhöhtes mütterliches Alter $>35$ Jahre

- Erhöhtes väterliches Alter

- Erhöhte Rate an Malformationen oder chromosomalen Aberrationen bei den Eltern

- Erhöhte Rate an Gestationsdiabetes

- Erhöhte Rate an präexistentem Diabetes mellitus

- Erhöhte Rate an Adipositas (BMI $\geq 30 \mathrm{~kg} / \mathrm{m}^{2}$ )

- Erhöhte Rate an Frühgeburten, Fehlgeburten oder Sektiones bei vorherigen Schwangerschaften

- Mehr Primiparae

- Toxinexposition? ${ }^{\mathrm{a}}$

- Ernährung? ${ }^{\mathrm{a}}$

${ }^{a}$ Diese Faktoren wurden bisher nicht untersucht.

Tabelle 2

Rate an chromosomalen Anomalien bei Kindern nach intrazytoplasmatischer Spermieninjektion (ICSI): Übersicht der wichtigsten Studienergebnisse

\section{Studie}

Bonduelle 1994

Bonduelle 1995

Testart 1996

Bonduelle 1996

Van Opstal 1997

Govaerts 1998

Loft 1999

Causio 1999

Van Golde 1999

Bonduelle 1999

Wennerholm 2000

Lam 2001

Alboughar 2001

Bonduelle 2002

Bonduelle 2002

\section{Anzahl untersuchter} ICSI-Kinder

43

100

115

486

71

101

209

63

57

1082

149

43

430

1437

1586

\section{Anomale Karyotypen [\%]}

0
0,99; postpartal nicht bestätigt

4,3

2,4

14

3,96

3,3

4,76

3,5

2,6

2,7

2,3

3,5

2,8

3,0 der Meiose gestört werden können [13]. Deshalb wird empfohlen, dass Paaren mit einer Schwangerschaft nach ICSI, ganz besonders bei Oligospermie des Vaters, eine invasive pränatale Diagnostik angeboten wird.

\section{Epigenetische Veränderungen nach assistierter Reproduktion}

Die genetische Information einer DNASequenz wird durch epigenetische Modifikationen (wie zum Beispiel DNA-Methylierung, Imprinting, kovalente Modi- fikationen von Histonen u.s.w.) vervollständigt. Dadurch kommt es zur Regulation der Genexpression. Diese sog. epigenetische Reprogrammierung findet mindestens an 2 kritischen Zeitpunkten statt, nämlich während der Gametogenese und während des embryonalen Präimplantationsstadiums [31].

Die Reprogrammierung während der Gametogenese ist zwingend notwendig für Imprinting-Mechanismen, welche die unterschiedliche Expression von väterlichen und mütterlichen Genen regulieren.
Wenn diese Mechanismen nun gestört werden, kommt es zu epigenetischen Veränderungen und damit zu einer Modifikation der DNA-Methylierung und/oder Chromatinstruktur, wodurch Änderungen der Genexpression und phänotypischer Merkmale resultieren. Überdies können epigenetische Veränderungen, welche kurz nach der Befruchtung, das heisst vor der Differenzierung in Keimzellen und somatische Zellen, auftreten, beide Zell-Linien betreffen und somit können diese epigenetischen Veränderungen dann von den Keimzellen weitervererbt werden.

Phänotypische Veränderungen durch Imprintingfehler sind eindeutig und früh diagnostizierbar, während Veränderungen durch Methylierungsdefekte viel subtiler sind, neurologische Entwicklungsverzögerungen oder geistige Behinderungen können darauf hinweisen. Ein Beispiel noch subtilerer phänotypischer Veränderungen sind Prädispositionen für Krebserkrankungen.

\section{( In-vitro-Kulturmedien können die Häufigkeit epigenetischer Veränderungen beeinflussen}

In neuerer Zeit wurde in mehreren Fallberichten auf eine Assoziation zwischen assistierter Reproduktionsmedizin und Imprintingstörungen wie zum Beispiel das Prader-Willi- oder Angelmann-Syndrom (AS; [15]) sowie das Beckwith-Wiedemann Syndrom (BWS) hingewiesen [16]. Und schon in den 199oer Jahren wurde an Mäuseembryonen gezeigt, dass durch die Zusammensetzung der In-vitro-Kulturmedien die Häufigkeit epigenetischer Veränderungen beeinflusst werden kann. Die genauen Mechanismen, durch welche ein Kulturmedium anomale epigenetische Modifikationen bewirken könnte, sind nicht bekannt.

Es wäre denkbar, dass Komponenten der Medien Methylgruppen auf DNA oder Histonen entfernen oder damit interagieren könnten. Möglich wäre auch, dass der Zeitpunkt der embryonalen Entwicklung durch die synthetischen Medien und Manipulation beeinträchtigt werden könnte, was wiederum zu einer Störung der epigenetischen Reprogrammierung und Genexpression führen würde. Wenn sich nun bewahrheiten würde, dass die In-vitro-Kultur ein Hauptrisikofaktor für die Entstehung 
von Imprintingfehlern wäre, würde durch die vermehrte Anwendung von Blastozystenkulturen (verlängerte Kulturen mit Transfer 5 Tage nach Befruchtung) eine größere Häufigkeit von Imprintingfehlern resultieren. Es müsste dabei nicht unbedingt zu den seltenen Syndromen wie BWS oder AS kommen, sondern es könnte sich bei diesen Imprintingfehlern auch um epigenetische Veränderungen handeln, die zurzeit noch gar nicht bekannt sind, die jedoch Auswirkungen auf die Langzeitgesundheit der IVF/ICSI-Kinder haben könnten.

Epigenetische Veränderungen werden als mögliche Ursache für die bisher nicht erklärte Häufung von intrauterinen Wachstumsretardierungen respektive niedrigerem Geburtsgewicht nach assistierter Reproduktionsmedizin diskutiert. Bisher konnten jedoch solche epigenetischen Veränderungen weder lokalisiert noch getestet werden, im Gegensatz beispielsweise zu den bekannten und nachweisbaren epigenetischen Veränderungen des BWS. Klinische Langzeit-Follow-up-Studien von Kindern nach IVF/ICSI sowie weitere molekulargenetische Forschung sind deshalb von größter Notwendigkeit, um diese Thesen zu widerlegen oder zu stützen.

\section{Fazit für die Praxis}

Das Risiko für chromosomale Anomalien ist bei Kindern nach In-vitro-Fertilisation (IVF) und intrazytoplasmatischer Spermieninjektion (ICSI) im Vergleich zu spontan gezeugten Kindern erhöht. Dies ergibt sich durch vererbte elterliche, aber auch durch De-novo auftretende chromosomale Anomalien. Deshalb sollte bei allen Paaren, bei denen eine IVF oder ICSI vorgesehen ist, eine Karyotypisierung durchgeführt werden. Im Falle einer ausgeprägten Oligospermie oder Azoospermie des Mannes sollte vor der ICSI-Therapie außerdem eine Abklärung auf zystische Fibrose sowie Mikrodeletionen vorgenommen werden. Zudem sollte Paaren mit einer Schwangerschaft nach ICSI, ganz besonders bei Oligospermie des Vaters, eine invasive pränatale Diagnostik angeboten werden.

Neue Ergebnisse zeigen ein leicht erhöhtes Risiko für Malformationen bei durch IVF und ICSI gezeugten Kindern. Für die Beratung von Kinderwunschpaaren er- gibt sich aus diesen Studienergebnissen, dass bei einer Spontankonzeption bei jeder 15. Schwangerschaft mit einer schweren Fehlbildung zu rechnen ist, nach ICSI bei jeder 12. Schwangerschaft. Paare müssen dementsprechend genauestens aufgeklärt werden.

Die Bedeutung epigenetischer Veränderungen nach Einsatz assistierter Reproduktionsmedizin ist bis heute noch unklar. Die Gesundheit dieser Kinder muss jedoch unbedingt in weiteren Studien ausgewertet werden.

\section{Korrespondierender Autor Dr. D. Wunder}

Universitätsfrauenklinik, Inselspital, Effingerstraße 102, 3010 Bern, Schweiz E-Mail: dorothea.wunder@insel.ch

Interessenkonflikt: Keine Angaben

\section{Literatur}

1. Alboughar H, Alboughar M, Mansour R, Serour G, Amin Y, Al-Inani H (2001) A prospective controlled study of karyotyping for 430 consecutive babies conceived through intracytoplasmic sperm injection. Fertil Steril 76: 249-253

2. Anthony S, Buitendijk SE, Dorrepaal, CA, Lindner K, Braat DD, den Ouden AL (2002) Congenital malformations in 4224 children conceived after IVF. Hum Reprod 17: 2089-2095

3. Beral V, Doyle P (1990) Report of the MRC working party on children conceived by in vitro fertilization. Births in Great Britain resulting from assisted conception, 1978-1987. BMJ 300: 1229-1233

4. Bergh T, Ericson A, Hillensjo T, Nygren KG, Wennerholm UB (1999) Deliveries and children born after in-vitro fertilisation in Sweden 1982-95: a retrospective cohort study. Lancet 354: 1579-1585

5. Bernardini L, Martini E, Geraedts JPM et al. (1997) Comparison of gonosomal aneuploidy in spermatozoa of normal fertile men and those with severe mal factor detected by in-situ hybridization. Mol Hum Reprod 3: 431-438

6. Bonduelle M, Legein J, Derde MP et al. (1995) Comparative follow-up study of 130 children born after intracytoplasmic sperm injection and 130 children born after in-vitro fertilization. Hum Reprod 10: 3327-3331

7. Bonduelle M, Libaers I, Deketelaere V et al. (2002) Neonatal data on a cohort of 2889 infants born after ICSI (1991-1999) and of 2995 infants born after IVF (1983-1999). Hum Reprod 17: 671-694

8. Bonduelle M, Desmyttere S, Buysse A et al. (1994) Prospective follow-up study of 55 children born after subzonal insemination and intracytoplasmic sperm injection. Hum Reprod 9: 1765-1769

9. Bonduelle M, Wilikens A, Buysse A et al. (1996) Prospective follow-up study of 877 children born after intracytoplasmic sperm injection (ICSI), with ejaculated, epididymal and testicular spermatozoa and after replacement of cryopreserved embryos obtained after ICSI. Hum Reprod 11: 131155
10. Bonduelle M, Legein J, Buysse A et al. (1996) Prospective follow-up study of 423 children born after intracytoplasmic sperm injection. Hum Reprod 11: 1558-1564

11. Bonduelle M, Von Assche E, Joris H et al. (2002) Prenatal testing in ICSI pregnancies: incidence of chromosomal anomalies in 1586 karyotypes and relation to sperm parameters. Hum Reprod 17: 2600 2614

12. Bonduelle M, Camus M, De Vos A et al. (1999) Seven years of intracytoplasmic sperm injection and follow-up of 1987 subsequent children. Hum Reprod 14: 243-264

13. Calogero AE, De Palma A, Grazioso C, Barone N, Romeo R, Rapazzo G, D’Agata R (2001) Aneuploidy rate in spermatozoa of selected men with abnormal semen parameters. Hum Reprod 16: 1172 1179

14. Colombero LT, Hariprashad JJ, Tsai MC, Rosenwaks Z, Palermo GD (1999) Incidence of sperm aneuploidy in relation to semen characteristics and assisted reproductive outcome. Fertil Steril 72: 90-96

15. Cox GF, Burger J, Lip V, Mau UA, Sperling K, Wu BL, Horsthemke B (2002) Intracytoplasmic sperm injection may increase the risk of imprinting defects. Am J Hum Genet 71: 162-164

16. DeBaun MR, Niemitz EL, Feinberg AP (2003) Association of in vitro fertilization with Beckwith-Wiedemann syndrome and epigenetic alterations of LIT1 and H19. Am J Hum Genet 72: 156-160

17. Ericson A, Kallen B (2001) Congenital malformations in infants born after IVF: a population-based study. Hum Reprod 16: 504-509

18. Govaerts I, Devreker F, Koenig I, Place I, Van den Bergh M, Englert Y (1998) Comparison of pregnancy outcome after intracytoplasmic sperm injection and in-vitro fertilization. Hum Reprod 13: 15141518

19. Greenland S, Ackermann DL (1995) Clomiphene citrate and neural tube defects: a pooled analysis of controlled epidemiologic studies and recommendations for future studies. Fertil Steril 64: 936-941

20. Hansen M, Kurinczuk JJ, Bower C, Webb S (2002) The risk of major birth defects after intracytoplasmic sperm injection and in vitro fertilization. $\mathrm{N}$ Engl J Med 346: 725-730

21. Johnson MD (1998) Genetic risks of intracytoplasmic sperm injection in the treatment of male infertility: recommendations for genetic counselling and screening. Fertil Steril 70: 397-411

22. Katalinic A, Rösch C, Ludwig M (2004) Pregnancy course and outcome after intracytoplasmic sperm injection: a controlled, prospective cohort study. Fertil Steril 81: 1604-1616

23. Koivurova S, Hartikainen AL, Gissler M, Hemmenki E, Sovio U, Jarvelin MR (2002) Neonatal outcome and congenital malformation in children born after in-vitro fertilization. Hum Reprod 17: 13911398

24. Ludwig M, Katalinic A (2002) Malformation rate in fetuses and children conceived after ICSI: results of a prospective cohort study. RBM online 5: 171-178

25. Nuoja-Huttunen S, Gissler M, Martikainen H, Tuomivaara $L$ (1999) Obstetric and perinatal outcome of pregnancies after intrauterine insemination. Hum Reprod 14: 2110-2115

27. Oliviennes F, Fanchin R, Lédée N, Righini C, Kadoch IJ, Frydmann R (2002) Perinatal outcome and development studies on children born after IVF. Hum Reprod Update 8: 117-128

28. Oliviennes F, Kerbrat $V$, Rufat $P$, Blanchet $V$, Fanchin R, Frydmann R (1997) Follow-up of a cohort of 422 children aged 6 to 13 years conceived by in vitro fertilization. Fertil Steril 67: 284-289 
29. Pandian Z, Bhattacharya S, Templeton A (2001) Review of unexplained infertility and obstetric outcome: a 10 year review. Hum Reprod 16: 2593-2597

30. Patrizio P (1995) Intracytoplasmic sperm injection (ICSI): potential genetic concerns. Hum Reprod 10: 2520-2523

31. Reik W, Dean W, Walter J (2001) Epigenetic reprogramming in mammalian development. Science 293: 1089-1093

32. Rufat $P$, Olivennes F, de Mouzon J, Dehan M, Frydman $R$ (1994) Task force report on the outcome of pregnancies and children conceived by in vitro fertilization (France 1987 to 1989). Fertil Steril 61: 324-330

33. Saunders K, Spensley J, Munro J, Halasz G (1996) Growth and physical outcome of children conceived by in vitro fertilization. Pediatrics 97: 688-692

34. van Loon K, Besseghir K, Eshkol A (1992) Neural tube defects after infertility treatment: a review. Fertil Steril 58: 875-884

35. Van Steirteghem A, Bonduelle M, Devroye P, Libaers I (2002) Follow-up of children after ICSI. Hum Reprod Update 8: 111-116

36. Sutcliffe AG, Taylor B, Saunders K, Thornton S, Liebermann BA, Grudzinskas JG (2001) Outcome in the second year of life after in-vitro fertilisation by intracytoplasmic sperm injection: a UK case-control study. Lancet 357: 2080-2084

37. Wennerholm UB, Bergh $C$, Hamberger L et al. (2000) Incidence of congenital malformations in children born after ICSI. Hum Reprod 15: 944-948

\section{„Der Gynäkologe“ bietet jeden Monat umfassende und aktuelle Beiträge zu interessanten Themenschwerpunkten aus allen Bereichen der Gynäkologie und Geburtshilfe.}

Möchten Sie ein bereits erschienenes Heft nachbestellen? Die folgenden Ausgaben können Sie direkt bei unserem Kundenservice zum Preis von je EUR 25,- beziehen:

\begin{tabular}{|c|c|c|}
\hline \multirow{2}{*}{ Der Gynäkologe } & \multicolumn{2}{|l|}{2004} \\
\hline & - Heft 1/04 & Risikomanagement \\
\hline E & - Heft 2/04 & Molekularbiologie und -genetik: \\
\hline$=$ & & Umsetzung in der Praxis \\
\hline & - Heft 3/04 & Gynäkologische Onkologie: \\
\hline & & Entwicklungen der molekularen Medizin \\
\hline & - Heft 4/04 & Geburtseinleitung \\
\hline & Heft 5/04 & Internistische Erkrankungen in der \\
\hline & & Schwangerschaft, Teil 2 \\
\hline$C^{\circ}$ & - Heft 6/04 & Schwangerschaft und gynäkologische Malignome \\
\hline$\cong$ & - Heft 7/04 & $\begin{array}{l}\text { Frauenärztliche Tätigkeit und } \\
\text { demographische Entwicklung }\end{array}$ \\
\hline & - Heft 8/04 & Forschung ermöglichen - Grenzen erkennen \\
\hline & & Kongressheft \\
\hline & - Heft 9/04 & Kinder- und Jugendgynäkologie \\
\hline & - Heft 10/04 & Update gynäkologische Onkologie \\
\hline 7 & - Heft 11/04 & Deszensus und Harninkontinenz \\
\hline & - Heft 12/04 & Akupunktur \\
\hline DerGyn & 2005 (Änderun & gen vorbehalten) \\
\hline$\equiv$ & - Heft 1/05 & Fehlbildungen \\
\hline-2 & - Heft 2/05 & Schwangerschaft und maligne Tumoren \\
\hline$=$ & - Heft 3/05 & Onkoplastische Chirurgie \\
\hline$=$ & - Heft 4/05 & Update Gynäkologische Endokrinologie \\
\hline$=$ & - Heft 5/05 & Update Mammakarzinom \\
\hline & - Heft 6/05 & Kontroversen in der Geburtshilfe \\
\hline & - Heft 7/05 & Beschwerden in der Schwangerschaft \\
\hline & - Heft 8/05 & HIV: Reproduktionsmedizin und Geburt \\
\hline & - Heft 9/05 & Arzneimitteltherapie in der Schwangerschaft \\
\hline & - Heft 10/05 & SLN in der Gynäkologie \\
\hline & - Heft 11/05 & Endoskopie \\
\hline & - Heft 12/05 & Altersgynäkologie \\
\hline
\end{tabular}

So erreichen Sie unseren Kundenservice:

\section{Springer Medizin Verlag GmbH}

Kundenservice Zeitschriften

Haberstr. 7

69126 Heidelberg

Tel.: +49 $6221345-4303$

Fax: +496221 345-4229

E-Mail:SDC-journals@springer-sbm.com 